\title{
ORIGINAL
}

ARTICLES

\section{Motivation for Participation in the American Board of Family Medicine Certification Program}

Michael R. Peabody, PhD; Lars E. Peterson, MD, PhD; Mingliang Dai, PhD; Aimee R. Eden, PhD, MPH; Elizabeth Hansen, BA; James C. Puffer, MD

BACKGROUND AND OBJECTIVES: Board certification programs have been criticized as not relevant to practice, not improving patient care, and creating additional burdens on already overburdened physicians. Many physicians may feel compelled to participate in board certification programs in order to satisfy employer, hospital, and insurer requirements; however, the influence of forces as motivators for physicians to continue board certification is poorly understood.

METHODS: We used data from the 2017 American Board of Family Medicine (ABFM) Family Medicine Certification Examination practice demographic registration questionnaire for those seeking to continue their certification, removing physicians who indicated they did not provide direct patient care. We utilized a mixed-methods design. For the quantitative analysis, a proportional odds logistic regression was used to examine the association between predictor variables and increasing levels of external motivation. For the qualitative analysis, we used a deductive approach to examine open-text responses.

RESULTS: Of the analytical sample of 7,545 family physicians, approximately one-fifth (21.4\%) were motivated to continue their board certification solely by intrinsic factors. Less than one-fifth (17.3\%) were motivated only by extrinsic factors, and the majority $(61.2 \%)$ reported mixed motivations for continuing their board certification. Only 38 respondents $(0.5 \%)$ included a negative opinion about the certification process in their open-text responses.

CONCLUSIONS: Approximately half of family physicians in this sample noted a requirement to continue their certification, suggesting that there has been no significant increase in the requirements from employers, credentialing bodies, or insurers for physicians to continue board certification noted in previously cited work. Furthermore, only $\mathbf{1 7 . 5 \%}$ of our sample reported solely external motivation to continue certification, indicating that real or perceived requirements are not the primary driver for most physicians to maintain certification.

(Fam Med. 2019;51(9):728-36.)

doi: 10.22454/FamMed.2019.850799
M edical professionalism has traditionally been defined using lists of shared values that professionals should exemplify, such as compassion, honesty, respect, and service. ${ }^{1}$ In 2012, the American Board of Medical Specialties (ABMS) Committee on Ethics and Professionalism adopted a definition of professionalism that highlights the primary function of professionalism in health care, namely, ensuring that physicians are worthy of patient and public trust. ${ }^{2}$ To help family physicians demonstrate their commitment to professionalism, the American Board of Family Medicine (ABFM) has created a continuing certification program that requires participants to meet rigorous standards established for professionalism, selfassessment and lifelong learning, improvement in practice, and maintaining their medical knowledge and clinical decision-making skills.

However, these types of continuing certification programs have been criticized by some physicians, who claim they are not relevant to practice, do not improve patient care, and create additional burdens on already overburdened physicians. ${ }^{3}$ It is unknown how widespread these

From the American Board of Family Medicine, Lexington, KY (Drs Peabody, Peterson, Dai, Eden, and Puffer, and Ms Hansen), and Department of Family and Community Medicine, University of Kentucky, Lexington, KY (Dr Peterson). 
sentiments are, and while many physicians feel compelled to participate in order to satisfy employer, hospital, and insurer requirements, ${ }^{3,4}$ the influence of external forces such as employer requirements as motivators for physicians to continue board certification is poorly understood.

Some evidence suggests that professional motivation is a significant factor in maintaining certification. A 2005 survey of general internists ${ }^{5}$ found that half of respondents reported participating in the American Board of Internal Medicine's (ABIM) Maintenance of Certification (MOC) program because it was required by one of their employers or payers; however, only one-third reported participation as required for employment, leading to speculation that perhaps participation was required by a secondary employer and not their primary source of income. The most common reasons for participating in ABIM's MOC program were to maintain professional image (59\%), update medical knowledge (51\%), and maintain or improve quality of patient care (45\%).

Furthermore, published evidence suggests that the consequences of losing certification-such as loss of employment or privileges-is not common. A 2005 study of hospital privileging for pediatricians found that $78 \%$ of hospitals did not require pediatricians to be board certified at the time of initial privileging. Only $45 \%$ required general pediatricians with time-limited certificates to recertify, and $69 \%$ of these allowed pediatricians to retain their privileges even after their certification expired, while $85 \%$ had never revoked or denied privileges due to failure to recertify. ${ }^{6} \mathrm{~A}$ follow-up study found that between 2005 and 2010, requiring certification for general pediatricians grew overall ( $67 \%$ to $80 \%)$, as well as for initial privileging ( $4 \%$ to $24 \%$ ), and more hospitals required recertification (33\% to $42 \%$ ). However, the rate of exceptions to certification policies became nearly universal (41\% to $99 \%$ ) and no significant change in proportion revoking or denying privileges due to loss of certification $(6 \%$ to $3 \%)$ was demonstrated. During this study period the ABMS MOC initiative had launched, but $89 \%$ of hospitals reported that they had not changed their board certification requirements as a result. ${ }^{7}$

A companion study of health plans in 2009 found that $90 \%$ did not require general pediatricians to be board certified at initial credentialing; $41 \%$ required general pediatricians to be certified at some point in their association with the plan; and $84 \%$ had never revoked or not renewed credentialing status for not recertifying. ${ }^{8}$ For surgeons, only 5\% of hospitals surveyed required certification at initial privileging; $12 \%$ restricted the privileges of nonboard-certified surgeons; and $82 \%$ allowed surgeons to retain privileges after their certification had expired. ${ }^{9}$

Given this information, we wished to examine family physicians' motivation for continuing $A B F M$ certification and to identify physician and practice characteristics associated with doing so.

\section{Methods}

\section{Data}

We used data from the $2017 \mathrm{ABFM}$ Family Medicine Certification Examination practice demographic registration questionnaire for those seeking to continue their certification. The questionnaire is a mandatory component of examination registration and is completed 3 to 4 months prior to examination day. The questionnaire asked about scope of practice, practice organization and location, practice ownership and size, as well as the applicant's motivation for continuing their certification. Each examination cohort of family physicians has been found to be largely representative and a reliable sample of the overall population of family physicians. ${ }^{10}$ The examination is administered twice per year and if physicians registered for both the spring and fall examinations, we kept their most recent responses. Physicians who did not provide direct patient care were excluded as they were not offered the question about motivation to continue their certification. We also restricted our sample to respondents who practiced primarily outpatient continuity care. Other demographic data were obtained from ABFM administrative databases.

\section{Variables}

Our primary outcome was the physicians' responses to the question, "Why are you seeking to continue your ABFM certification at this time?" The question was a select-allthat-apply format with 11 possible responses, including an "other" option with free-text responses. Freetext responses were omitted from the regression model and analyzed using qualitative methods only. We calculated our dependent variable based on the combination of responses to this survey question. The physician was identified as extrinsically motived if only one or more of the following three options were selected: Required by my employer; Required for hospital privileges/credentialing; and/or Required by one or more payer/insurance company. The physician was identified as intrinsically motivated if only one or more of the following seven options were selected: Maintain professional image; Personal preference; Professional advancement; Maintain or improve patient satisfaction; Patients prefer being treated by board certified physician; Certification program helps me update my medical knowledge; and/or certification program helps me monitor or improve the quality of my patient care. Selections from each of the intrinsic and extrinsic responses resulted in the physician being classified as having mixed motivation.

We adapted Herzberg's motivation-hygiene theory ${ }^{11,12}$ for our classification system. Herzberg's theory posits that extrinsic motivators exist outside an individual (eg, money and status) while intrinsic motivators exist within an individual with effort linked to the level of interest in the work being performed (eg, purpose, responsibility, and learning). 
Variables on physician characteristics included physician age, scope of practice, race, ethnicity, gender, and US or international medical school graduate. Practice characteristic variables included ownership, size, specialty mix, faculty status, hours per week in direct patient care, years in practice, and practice type.

\section{Analysis}

We preformed adjusted and unadjusted quantitative analyses. For the unadjusted analyses, we used $\chi^{2}$ tests for categorical variables and $t$ tests for continuous variables. For the adjusted analysis we used a proportional odds logistic regression, which provides a common odds ratio (OR) for increasing from one level to the next. For our analysis, the levels increased in order from intrinsic, to mixed, to extrinsic. The covariates included race, ethnicity, gender, practice site ownership status, practice site size, practice site specialty, faculty status, hours providing direct patient care, years in practice, primary practice site type, and individual scope of practice (ISOP ${ }^{13}$ ) score. All analyses were conducted using $R$ 3.3.1 (R Project for Statistical Computing, R Foundation; http://www.rproject.org/). Unadjusted analyses used the compareGroups package ${ }^{14}$ and the adjusted analysis used the proportional odds logistic regression (polr) function in the MASS package. ${ }^{15}$

For the qualitative analysis, we used a deductive approach to analyze open-text responses to the "other" option. First, after reading all opentext comments, we coded preexisting multiple-choice answers when applicable (eg, "Keep knowledge up to date" was coded as "Certification program helps me update my medical knowledge"). We assigned remaining open-text answers to new, mutually exclusive categories through an iterative process. Nonsensical and nonapplicable responses were discarded. We also assessed open-text responses for the presence of negative or positive attitude about continuous certification. The American Academy of Family Physicians' Institutional Review Board approved this study.

\section{Results}

There were 11,157 registrations for the ABFM Family Medicine Certification Examination in 2017. Of these, 1,008 registered for both the spring and fall examination and only the most recent response was retained. We further removed respondents who did not practice direct patient care $(n=590)$ or outpatient continuity care $(\mathrm{n}=1979)$. Thirtyfive physicians who only answered "other" to the question on reasons why they were seeking to continue their certification were removed from quantitative analysis but retained in the qualitative analysis. Our final

Table 1: Reasons of Family Physicians Seeking to Continue Their American Board of Family Physicians Certification in 2017 ( $N=7,545)$

\begin{tabular}{|l|c|c|}
\hline \multicolumn{1}{|c|}{ Extrinsic } & N & $\%$ \\
\hline Required by my employer & & 50.0 \\
\hline Required for hospital privileges/credentialing & 3,774 & 56.1 \\
\hline Required by one or more payer/insurance company & 4,231 & 45.6 \\
\hline \multicolumn{1}{|c|}{ Intrinsic } & 3,438 & \\
\hline Maintain professional image & & 54.8 \\
\hline Personal preference & 4,136 & 52.5 \\
\hline Professional advancement & 3,963 & 35.4 \\
\hline Maintain or improve patient satisfaction & 2,673 & 25.0 \\
\hline Patients prefer being treated by board certified physicians & 1,890 & 36.8 \\
\hline Certification program helps me update my medical knowledge & 2,778 & 51.7 \\
\hline Certification program helps me monitor or improve the quality of my patient care & 3,904 & 42.1 \\
\hline & 3,173 & \\
\hline Other (please specify) & & \\
\hline & 192 & 2.5 \\
\hline Extrinsic only responses & & \\
\hline Intrinsic only responses & 1,309 & 17.3 \\
\hline Mixed extrinsic and intrinsic responses & 1,616 & 21.4 \\
\hline
\end{tabular}

Note: $\mathrm{N}$ is the number of responses to the `select all that apply question and \% is the number of responses divided by the number of physicians in the sample. 
analytical sample included 7,545 family physicians.

Physicians gave varying reasons for maintaining certification (Table 1), the most common response being an extrinsic motivation, "Required for hospital privileges/credentialing." The next three most popular responses were intrinsic motivations, including "Maintain professional image," "Personal preference," and "Certification program helps me update my medical knowledge." Overall, only $17 \%$ selected only extrinsic reasons.

Our sample had a mean (SD) age of 51.7 (8.9) years, were mostly male
(56.6\%), white (72.2\%), non-Hispanic (93.1\%), and USMGs (78.0\%; Table 2). A majority (63.0\%) had no official ownership stake in their practice; one-third (34.9\%) described their primary practice site as a private practice; a majority (52.0\%) described their site specialty as family medicine only; and $39.0 \%$ spent 40 or more hours per week in direct patient care.

There were statistically significant differences in the unadjusted analyses $(P<.05)$ between the physicians' motivation to pursue ABFM certification by race, ethnicity, gender, medical school training, practice site ownership, practice site size, faculty status, hours in direct patient care, years in practice, primary practice site type, and scope of practice.

In adjusted models we found that identifying as Asian (OR $=0.79$, $\mathrm{CI}=0.68,0.92)$ or black $(\mathrm{OR}=0.55$, $\mathrm{CI}=0.45,0.67)$ was associated with lower odds of extrinsic motivation compared to identifying as white (Table 3). Working 0 to 8 hours per week $(\mathrm{OR}=0.69, \mathrm{CI}=0.53,0.89)$ and 17 to 24 hours per week in direct patient care $(\mathrm{OR}=0.82, \mathrm{CI}=0.70,0.97)$ was associated with lower odds of extrinsic motivation compared to working 40 or more hours per week in

Table 2: Physician and Practice Characteristics by Motivation to Continue American Board of Family Medicine Certification

\begin{tabular}{|c|c|c|c|c|c|}
\hline & [ALL] & Intrinsic & Mixed & Extrinsic & $\boldsymbol{P}$ \\
\hline & $\mathrm{N}=7,546$ & $\mathrm{~N}=1,616$ & $\mathrm{~N}=4,621$ & $\mathrm{~N}=\mathbf{1 , 3 0 9}$ & \\
\hline \multicolumn{6}{|l|}{ Physician Characteristics } \\
\hline Age & $51.7(8.98)$ & $51.4(9.20)$ & $51.8(9.07)$ & $51.6(8.35)$ & .269 \\
\hline Scope of practice (ISOP) & $13.2(3.72)$ & $12.3(3.70)$ & $13.5(3.66)$ & $13.1(3.75)$ & $<.001$ \\
\hline Race & & & & & $<.001$ \\
\hline American Indian or Alaska Native & $69(0.91 \%)$ & $17(1.05 \%)$ & $38(0.82 \%)$ & $14(1.07 \%)$ & \\
\hline Asian & $1,112(14.7 \%)$ & $320(19.8 \%)$ & $664(14.4 \%)$ & $128(9.78 \%)$ & \\
\hline Black or African American & $456(6.04 \%)$ & $150(9.28 \%)$ & $257(5.56 \%)$ & $49(3.74 \%)$ & \\
\hline Native Hawaiian or Other Pacific Islander & $36(0.48 \%)$ & $10(0.62 \%)$ & $21(0.45 \%)$ & $5(0.38 \%)$ & \\
\hline Other & $427(5.66 \%)$ & $121(7.49 \%)$ & $216(4.67 \%)$ & $90(6.88 \%)$ & \\
\hline White & $5,446(72.2 \%)$ & $998(61.8 \%)$ & $3,425(74.1 \%)$ & $1,023(78.2 \%)$ & \\
\hline Ethnicity & & & & & $<.001$ \\
\hline Hispanic or Latino & $523(6.93 \%)$ & $156(9.65 \%)$ & $280(6.06 \%)$ & $87(6.65 \%)$ & \\
\hline Non-Hispanic & $7,023(93.1 \%)$ & $1,460(90.3 \%)$ & $4,341(93.9 \%)$ & $1,222(93.4 \%)$ & \\
\hline Gender & & & & & .019 \\
\hline Female & $3,278(43.4 \%)$ & $709(43.9 \%)$ & $2,046(44.3 \%)$ & $523(40.0 \%)$ & \\
\hline Male & $4,268(56.6 \%)$ & $907(56.1 \%)$ & $2,575(55.7 \%)$ & $786(60.0 \%)$ & \\
\hline Medical School Training & & & & & $<.001$ \\
\hline IMG & $1,659(22.0 \%)$ & $531(33.0 \%)$ & $945(20.5 \%)$ & $183(14.0 \%)$ & \\
\hline USMG & $5,871(78.0 \%)$ & $1,080(67.0 \%)$ & $3,669(79.5 \%)$ & $1,122(86.0 \%)$ & \\
\hline \multicolumn{6}{|l|}{ Practice Characteristics } \\
\hline Primary Practice Ownership & & & & & $<.001$ \\
\hline Sole owner & $1,044(13.8 \%)$ & $344(21.3 \%)$ & $561(12.1 \%)$ & $139(10.6 \%)$ & \\
\hline $\begin{array}{l}\text { Self-employed as a contractor (including } \\
\text { locums) }\end{array}$ & $238(3.15 \%)$ & $82(5.07 \%)$ & $124(2.68 \%)$ & $32(2.44 \%)$ & \\
\hline Partial owner or shareholder & $1,423(18.9 \%)$ & $292(18.1 \%)$ & $905(19.6 \%)$ & $226(17.3 \%)$ & \\
\hline
\end{tabular}

(continued on next page) 
Table 2, continued

\begin{tabular}{|c|c|c|c|c|c|}
\hline Practice Characteristics & [ALL] & Intrinsic & Mixed & Extrinsic & $\boldsymbol{P}$ \\
\hline Primary Practice Ownership & $\mathrm{N}=7,546$ & $\mathrm{~N}=1,616$ & $\mathrm{~N}=4,621$ & $\mathrm{~N}=\mathbf{1 , 3 0 9}$ & \\
\hline $\begin{array}{l}\text { No official ownership stake (100\% } \\
\text { employed) }\end{array}$ & $4,751(63.0 \%)$ & $864(53.5 \%)$ & $2,985(64.6 \%)$ & $902(68.9 \%)$ & \\
\hline Other & $90(1.19 \%)$ & $34(2.10 \%)$ & $46(1.00 \%)$ & $10(0.76 \%)$ & \\
\hline Primary Practice Site Size & & & & & $<.001$ \\
\hline Solo practice & $944(12.5 \%)$ & $292(18.1 \%)$ & $516(11.2 \%)$ & $136(10.4 \%)$ & \\
\hline 2-5 providers & $2,615(34.7 \%)$ & $625(38.7 \%)$ & $1,544(33.4 \%)$ & $446(34.1 \%)$ & \\
\hline $6-20$ providers & $2,317(30.7 \%)$ & $428(26.5 \%)$ & $1,484(32.1 \%)$ & $405(30.9 \%)$ & \\
\hline$>20$ providers & $1,670(22.1 \%)$ & $271(16.8 \%)$ & $1,077(23.3 \%)$ & $322(24.6 \%)$ & \\
\hline Primary Practice Specialty & & & & & .292 \\
\hline Family medicine only & $3,923(52.0 \%)$ & $833(51.5 \%)$ & $2,407(52.1 \%)$ & $683(52.2 \%)$ & \\
\hline Multiple specialties & $1,581(21.0 \%)$ & $317(19.6 \%)$ & $976(21.1 \%)$ & $288(22.0 \%)$ & \\
\hline Primary care specialty mix (FM, IM, PED) & $2,042(27.1 \%)$ & $466(28.8 \%)$ & $1,238(26.8 \%)$ & $338(25.8 \%)$ & \\
\hline Faculty & & & & & $<.001$ \\
\hline Yes, core/salaried faculty & $742(9.83 \%)$ & $86(5.32 \%)$ & $499(10.8 \%)$ & $157(12.0 \%)$ & \\
\hline Yes, volunteer/clinical faculty & $1,698(22.5 \%)$ & $305(18.9 \%)$ & $1,102(23.8 \%)$ & $291(22.2 \%)$ & \\
\hline No & $5,106(67.7 \%)$ & $1,225(75.8 \%)$ & $3,020(65.4 \%)$ & $861(65.8 \%)$ & \\
\hline Hours per Week Direct Patient Care & & & & & $<.001$ \\
\hline $0-8$ & $271(3.59 \%)$ & $66(4.08 \%)$ & $172(3.72 \%)$ & $33(2.52 \%)$ & \\
\hline $9-16$ & $465(6.16 \%)$ & $86(5.32 \%)$ & $289(6.25 \%)$ & $90(6.88 \%)$ & \\
\hline $17-24$ & $799(10.6 \%)$ & $168(10.4 \%)$ & $512(11.1 \%)$ & $119(9.09 \%)$ & \\
\hline $25-32$ & $1,709(22.6 \%)$ & $342(21.2 \%)$ & $1,049(22.7 \%)$ & $318(24.3 \%)$ & \\
\hline $33-40$ & $1,356(18.0 \%)$ & $249(15.4 \%)$ & $880(19.0 \%)$ & $227(17.3 \%)$ & \\
\hline $40+$ & $2,946(39.0 \%)$ & $705(43.6 \%)$ & $1,719(37.2 \%)$ & $522(39.9 \%)$ & \\
\hline Years in Practice & & & & & $<.001$ \\
\hline $0-10$ & $1,985(26.3 \%)$ & $485(30.0 \%)$ & $1211(26.2 \%)$ & $289(22.1 \%)$ & \\
\hline $11-20$ & $2,546(33.7 \%)$ & $551(34.1 \%)$ & $1,512(32.7 \%)$ & $483(36.9 \%)$ & \\
\hline $21-30$ & $2124(28.1 \%)$ & $385(23.8 \%)$ & $1,331(28.8 \%)$ & $408(31.2 \%)$ & \\
\hline $30+$ & $891(11.8 \%)$ & $195(12.1 \%)$ & $567(12.3 \%)$ & $129(9.85 \%)$ & \\
\hline Primary Practice Type & & & & & $<.001$ \\
\hline Academic medical center & $515(6.82 \%)$ & $58(3.59 \%)$ & $340(7.36 \%)$ & $117(8.94 \%)$ & \\
\hline Veterans affairs & $397(5.26 \%)$ & $147(9.10 \%)$ & $196(4.24 \%)$ & $54(4.13 \%)$ & \\
\hline Safety net & $722(9.57 \%)$ & $167(10.3 \%)$ & $441(9.54 \%)$ & $114(8.71 \%)$ & \\
\hline Hosp/owned & $2,505(33.2 \%)$ & $331(20.5 \%)$ & $1,643(35.6 \%)$ & $531(40.6 \%)$ & \\
\hline Private practice & $2,633(34.9 \%)$ & $718(44.4 \%)$ & $1,540(33.3 \%)$ & $375(28.6 \%)$ & \\
\hline HMO & $406(5.38 \%)$ & $84(5.20 \%)$ & $267(5.78 \%)$ & $55(4.20 \%)$ & \\
\hline Other & $368(4.88 \%)$ & $111(6.87 \%)$ & $194(4.20 \%)$ & $63(4.81 \%)$ & \\
\hline
\end{tabular}

Notes: Sixteen missing medical school training.

Abbreviations: CI, confidence interval; OR, odds ratio; FM, family medicine; IM internal medicine; PEDS, pediatrics; HMO, health maintenance organization; USMG, United States medical graduate; IMG, international medical graduate; ISOP, individual scope of practice. 
Table 3: Proportional Odds of Motivation for Participation in Continuing Certification for Family Physicians

\begin{tabular}{|c|c|c|}
\hline Variable & OR & (95\% Cl) \\
\hline \multicolumn{3}{|l|}{ Physician Characteristics } \\
\hline Age, per year increase & 1.00 & $(0.99,1.01)$ \\
\hline Scope of practice, per one unit increase in ISOP & 1.00 & $(0.99,1.01)$ \\
\hline \multicolumn{3}{|l|}{ Race } \\
\hline White & ref & \\
\hline American Indian or Alaska Native & 0.86 & $(0.53,1.40)$ \\
\hline Asian & 0.79 & $(0.68,0.92)$ \\
\hline Black or African American & 0.55 & $(0.45,0.67)$ \\
\hline Native Hawaiian or other Pacific Islander & 0.75 & $(0.38,1.46)$ \\
\hline Other & 1.05 & $(0.85,1.30)$ \\
\hline \multicolumn{3}{|l|}{ Ethnicity } \\
\hline Hispanic & ref & \\
\hline Non-Hispanic & 1.23 & $(1.01,1.49)$ \\
\hline \multicolumn{3}{|l|}{ Gender } \\
\hline Female & ref & \\
\hline Male & 1.03 & $(0.93,1.14)$ \\
\hline \multicolumn{3}{|l|}{ Medical School Training } \\
\hline IMG & ref & \\
\hline USMG & 1.67 & $(1.46,1.91)$ \\
\hline \multicolumn{3}{|l|}{ Practice Characteristics } \\
\hline \multicolumn{3}{|l|}{ Primary Practice Ownership } \\
\hline Sole owner & ref & \\
\hline No official ownership stake (100\% employed) & 1.35 & $(1.07,1.70)$ \\
\hline Other & 0.76 & $(0.48,1.22)$ \\
\hline Partial owner or shareholder & 1.32 & $(1.07,1.64)$ \\
\hline Self-employed as a contractor (including locums) & 0.90 & $(0.66,1.24)$ \\
\hline \multicolumn{3}{|l|}{ Primary Practice Site Size } \\
\hline Solo practice & ref & \\
\hline 2-5 providers & 0.90 & $(0.74,1.09)$ \\
\hline $6-20$ providers & 1.01 & $(0.81,1.26)$ \\
\hline$>20$ providers & 1.19 & $(0.94,1.51)$ \\
\hline \multicolumn{3}{|l|}{ Practice Site Specialty } \\
\hline Family medicine only & ref & \\
\hline Multiple specialties (not only primary care) & 0.97 & $(0.84,1.11)$ \\
\hline Primary care specialty mix (FM, IM, PEDS) & 1.02 & $(0.91,1.14)$ \\
\hline \multicolumn{3}{|l|}{ Faculty } \\
\hline No & ref & \\
\hline Yes, core/salaried faculty & 1.32 & $(1.07,1.63)$ \\
\hline Yes, volunteer/clinical faculty & 1.08 & $(0.96,1.21)$ \\
\hline
\end{tabular}

(continued on next page) 
Table 3: Continued

\begin{tabular}{|c|c|c|}
\hline Variable & OR & $(95 \% \mathrm{Cl})$ \\
\hline \multicolumn{3}{|c|}{ Practice Characteristics } \\
\hline \multicolumn{3}{|l|}{ Hours per Week Direct Patient Care } \\
\hline $40+$ & ref & \\
\hline $0-08$ & 0.69 & $(0.53,0.89)$ \\
\hline $9-16$ & 0.89 & $(0.72,1.10)$ \\
\hline $17-24$ & 0.82 & $(0.70,0.97)$ \\
\hline $25-32$ & 1.02 & $(0.90,1.15)$ \\
\hline $33-40$ & 1.04 & $(0.91,1.18)$ \\
\hline \multicolumn{3}{|l|}{ Years in Practice } \\
\hline $0-10$ & ref & \\
\hline $11-20$ & 1.21 & $(1.05,1.39)$ \\
\hline $21-30$ & 1.17 & $(0.96,1.43)$ \\
\hline $30+$ & 0.86 & $(0.65,1.14)$ \\
\hline \multicolumn{3}{|l|}{ Primary Practice Type } \\
\hline Private practice & ref & \\
\hline Academic medical center & 1.63 & $(1.23,2.18)$ \\
\hline HMO & 1.18 & $(0.94,1.49)$ \\
\hline Hospital/owned & 1.72 & $(1.46,2.02)$ \\
\hline Other & 1.00 & $(0.78,1.28)$ \\
\hline Safety net & 1.09 & $(0.88,1.34)$ \\
\hline Veterans affairs & 0.66 & $(0.51,0.85)$ \\
\hline
\end{tabular}

Abbreviations: CI, confidence interval; OR, odds ratio; FM, family medicine; IM internal medicine; PEDS, pediatrics; HMO, health maintenance organization; USMG, United States medical graduate; IMG, international medical graduate; ISOP, individual scope of practice; ref, reference group for analytic comparison.

* Increasing odds ratio indicates increasing levels of extrinsic motivation.

direct patient care. Working at Veterans Affairs was associated with lower odds of extrinsic motivation compared to private practice physicians $(\mathrm{OR}=0.66, \mathrm{CI}=0.51,0.85)$.

Furthermore, variables associated with greater odds of extrinsic motivation included non-Hispanic compared to Hispanic $(\mathrm{OR}=1.23$, $\mathrm{CI}=1.01,1.49$ ); core/salaried faculty compared to nonfaculty $(\mathrm{OR}=1.32$, $\mathrm{CI}=1.07,1.63)$; USMG compared to IMG (OR=1.67, $\mathrm{CI}=1.46,1.91)$; academic medical center $(\mathrm{OR}=1.63$, $\mathrm{CI}=1.23,2.18)$ and hospital/owned ( $\mathrm{OR}=1.72, \mathrm{CI}=1.46,2.02)$ compared to private practice; no ownership stake $(\mathrm{OR}=1.35, \mathrm{CI}=1.07,1.70)$ and part-owner/shareholder $(\mathrm{OR}=1.32$, $\mathrm{CI}=1.07,1.64)$ compared to sole owner; and 11 to 20 years in practice compared to 0 to 10 years in practice $(\mathrm{OR}=1.21, \mathrm{CI}=1.05,1.39)$.

While 238 respondents indicated an "other" reason for maintaining certification, 16 were removed because they were nonmeaningful (eg, "n/a"), and 30 were removed because they fit one of the listed response options (of these, 17 were extrinsic and 13 were intrinsic motivations). Fewer than $2 \%(\mathrm{n}=192)$ provided novel open-text answers. Most of these were extrinsic motivations, including required to maintain subspecialty certification $(n=38)$, industry expectation/general requirement $(n=31)$, and financial incentive/increased pay $(n=17)$. Just 27 provided other intrinsic motivations for maintaining certification, the most common category being the maintenance of professional identity $(\mathrm{n}=13)$. Only 38 respondents $(0.5 \%)$ included a negative opinion about the certification process in their open-text responses.

\section{Discussion}

In a large representative sample of family physicians seeking to continue their ABFM certification, we found that one-fifth $(21.4 \%)$ were motivated to continue their board certification solely by intrinsic factors, less than one-fifth (17.3\%) were motivated only by extrinsic factors, and the vast majority $(62.1 \%)$ reported mixed motivations for continuing their board certification. These findings provide the first real data in nearly 10 years on the prevalence of requirements for board certification and physicians' reasons for 
continuing certification. Importantly, our findings place in context the anecdotes of physicians being required to continue certification as over half report these requirements, but a majority also report intrinsic motivations for holding themselves to the high standards of board certification. Furthermore, our results largely mirror those from the 2005 survey of general internists, suggesting that there has not been a significant increase in the requirements from employers, credentialing bodies, or insurers for physicians to be board certified. ${ }^{5}$

Herzberg's motivation-hygiene theory provides a framework for examining the motivational factors related to continuing certification. While not capturing all the subtleties that drive an individual's motivation, the framework is useful for understanding the larger factors involved. Our results identified several factors associated with intrinsic motivation for continuing certification, including working less than full time in patient care and working for Veterans Affairs. It is likely that some physicians who work only part time may be exempt from credentialing requirements related to specialty board certification.

Factors related to extrinsic motivation included core/salaried faculty status, primary practice type of Academic Medical Center or Hospital, and no practice ownership stake or part-time shareholder. Physicians who are core faculty, work at academic medical centers or hospitals, or have no ownership stake in their practice are subject to requirements placed upon them by outside entities. In these arrangements the physician may have a lower level of control over their work environment and requirements. With physicians rapidly moving to employed models over the last decade, ${ }^{16}$ this may explain the differences between our findings and those from a decade ago. Future research utilizing cohort studies of physicians who lost certification or new surveys of employers and credentialers is needed to help better understand this issue.

It is important to note that the physicians in this analysis were those who had a current ABFM certification and were continuing their certification; therefore, the primary research question was "Why are you continuing?" rather than "Would you continue if you were not required?" This second question presumes that all physicians in the sample were somehow required to continue their certification and the results show that this is clearly not the case. However, the question of whether those who are required would continue if they were not required does deserve consideration and future research should examine this issue. Therefore, future versions of this survey will have a follow-up question added for those physicians who only respond that they are required to recertify by asking "If you were not required to participate, would you certify?"

\section{Limitations}

This research has some limitations. We studied only one medical specialty and the results may vary based on specialty type. Also, because the question was a "Select all that apply" type, we are unable to determine causality and/or the primary driving force behind a physician's motivation to continue certification. It is also possible that a social desirability bias exists as the board holding the physician's certification is asking whether the physician values the certification. However, given the distribution of responses (ie, 17\% provided extrinsic-only responses), the potential impact of this bias on the results is likely small. It is also important to note the potential for bias in our interpretation since all authors were employees of the ABFM at the time this research was conducted. However, certifying boards have a duty to evaluate their programs for effectiveness and burden; subjecting their findings to peer-review serves as a check on potential bias. Finally, some physicians may be unaware of their employment requirements regarding board certification and may believe there are requirements when none exist, or may believe there are not requirements when in fact there are.

\section{Conclusion}

Approximately half of family physicians noted a requirement to continue their certification, suggesting that there has been no significant increase in the requirements from employers, credentialing bodies, or insurers for physicians to continue board certification noted in previously cited work. Furthermore, only $17.3 \%$ of our sample reported solely external motivation to continue certification, indicating that real or perceived requirements are not the primary driver for most physicians to continue certification.

CONFLICTS OF INTEREST: All authors are employees of American Board of Family Medicine.

CORRESPONDING AUTHOR: Address correspondence to Dr Michael R. Peabody, 1648 McGrathiana Pkwy, Suite 550, Lexington, KY 40511. 859-269-5626. mpeabody@theabfm.org.

\section{References}

1. Wynia MK, Papadakis MA, Sullivan WM Hafferty FW. More than a list of values and desired behaviors: a foundational understanding of medical professionalism. Acad Med. 2014;89(5):712-714.

2. Hafferty FW, Papadakis MA, Sullivan W, Wynia MK. The American Board of Medical Specialties Ethics and Professionalism Committee Definition of Professionalism. Chicago, IL: American Board of Medical Specialties; 2012.

3. Teirstein PS. Boarded to death-why maintenance of certification is bad for doctors and patients. N Engl J Med. 2015;372(2):106-108.

4. Cook DA, Blachman MJ, West CP, Wittich CM. Physician Attitudes About Maintenance of Certification: A Cross-Specialty National Survey. Mayo Clin Proc. 2016;91(10):1336-1345.

5. Lipner RS, Bylsma WH, Arnold GK, Fortna GS, Tooker J, Cassel CK. Who is maintaining certification in internal medicine-and why? A national survey 10 years after initial certification. Ann Intern Med. 2006;144(1):29-36.

6. Freed GL, Uren RL, Hudson EJ, Lakhani I, Wheeler JR, Stockman JA III; Research Advisory Committee of the American Board of Pediatrics. Policies and practices related to the role of board certification and recertification of pediatricians in hospital privileging. JAMA. 2006;295(8):905-912. 
7. Freed GL, Dunham KM, Gebremariam A. Changes in hospitals' credentialing requirements for board certification from 2005 to 2010. J Hosp Med. 2013;8(6):298-303.

8. Freed GL, Singer D, Lakhani I, Wheeler JR, Stockman JA III; Research Advisory Committee of the American Board of Pediatrics. Use of board certification and recertification of pediatricians in health plan credentialing policies. JAMA. 2006;295(8):913-918.

9. Freed GL, Dunham KM, Singer D. Use of board certification and recertification in hospital privileging: policies for general surgeons, surgical specialists, and nonsurgical subspecialists. Arch Surg. 2009;144(8):746-752.
10. Peterson LE, Fang B, Phillips RL Jr, Avant R, Puffer JC. The American Board of Family Medicine's Data Collection Method for Tracking Their Specialty. J Am Board Fam Med. 2019;32(1):89-95.

11. Herzberg F. One more time: how do you motivate employees? 1968. Harv Bus Rev. 2003;81(1):87-96.

12. Herzer KR, Pronovost PJ. Physician Motivation: Listening to What Pay-for-Performance Programs and Quality Improvement Collaboratives Are Telling Us. Jt Comm J Qual Patient Saf. 2015;41(11):522-528.
13. O’Neill T, Peabody MR, Blackburn BE, Peterson LE. Creating the Individual Scope of Practice (I-SOP) scale. J Appl Meas. 2014;15(3):227239.

14. Subirana I, Sanz H, Vila J. Building bivariate tables: the compareGroups Package for R. J Stat Softw. 2014;57(12):1-16

15. Venables WN, Ripley BD. Modern Applied Statistics with S. 4th ed. New York: Springer; 2002.

16. Kane CK. Updated Data on Physician Practice Arrangements: Physician Ownership Drops Below 50 Percent. Chicago: American Medical Association; 2017. 